\title{
APRENDIZAGEM COLABORATIVA NO ENSINO DE ENGENHARIA: UMA CORRELAÇÃO ENTRE O NÍVEL DE COLABORAÇÃO E DESEMPENHO ACADÊMICO
}

\author{
COLLABORATIVE LEARNING IN ENGINEERING TEACHING: A CORRELATION BETWEEN \\ LEVEL OF COLLABORATION AND ACADEMIC PERFORMANCE
}

Julia Eunice Fernandes ${ }^{1}$, Caroline Subirá Pereira² ${ }^{2}$ Eliana Cláudia Mayumi Ishikawa ${ }^{3}$, Guataçara dos Santos Junior ${ }^{4}$, Simone Nasser Matos ${ }^{5}$

DOI: $10.37702 / R E E 2236-0158 . v 39 p 79-88.2020$

\section{RESUMO}

O objetivo deste artigo é analisar estatisticamente a correlação entre as duas principais variáveis envolvidas em uma pesquisa de doutorado: o nível de colaboração e o desempenho do acadêmico na visão do professor. A aplicação aconteceu na disciplina de Probabilidade e Estatística, no curso de Engenharia de Produção de uma Universidade Tecnológica Federal. A quantificação do nível de colaboração do acadêmico foi possível por meio do Objeto Virtual de Aprendizagem denominado Collabora (ISHIKAWA, 2018), que permite maior agilidade no processo de obtenção de dados referentes à aprendizagem colaborativa. Para atingir o objetivo proposto aplicou-se conhecimentos estatísticos de Correlação e Regressão Linear, com os quais foi possível calcular o coeficiente e analisar o quão significativa é a correlação entre as variáveis. Além disso, foi possível construir um modelo que representa estatisticamente essa correlação. Os resultados quantitativos mostraram uma correlação significativa entre o nível de colaboração do futuro engenheiro comparado com o desempenho acadêmico, classificada entre moderada e forte, indicando que quanto maior a colaboração do aluno para as atividades realizadas no Collabora, melhor é o seu desempenho na disciplina, constatando-se, assim, a eficiência da aprendizagem colaborativa.

Palavras-chave: aprendizagem colaborativa; desempenho acadêmico; formação de engenheiros.

\begin{abstract}
The purpose of this article is to statistically analyze the correlation between the two main variables involved in a doctoral research: the level of collaboration and the academic performance in the teacher's view. The application happened in the discipline of Probability and Statistics, in the Production Engineering course in a Federal Technological University. The quantification of the level of collaboration of the academic was possible through the Virtual Learning Object called Collabora (ISHIKAWA, 2018), which allows greater agility in the process of obtaining data regarding collaborative learning. In order to reach the proposed objective, we applied statistical knowledge of Correlation and Linear Regression, which made it possible to calculate the coefficient and to analyze how significant the correlation between the variables was. In addition, it was possible to construct a model that statistically represents this correlation. The quantitative results showed a significant correlation between the level of collaboration of the future engineer compared to the academic performance, classified as moderate and strong, indicating that the greater the collaboration of the

1 Bolsista CNPq de Iniciação Científica do Programa de Ensino de Ciência e Tecnologia, Universidade Tecnológica Federal do Paraná - UTFPR Ponta Grossa. juliafernandes@alunos.utfpr.edu.br

2 Doutoranda, bolsista CAPES, do Programa de Pós-Graduação em Ensino de Ciência e Tecnologia, da Universidade Tecnológica e Federal do Paraná

- UTFPR - Ponta Grossa. carolinepereira@alunos.utfpr.edu

3 Professora titular da Universidade Tecnológica Federal do Paraná - UTFPR - Ponta Grossa. ecm.ishikawa50@gmail.com

4 Professor da Universidade Tecnológica Federal do Paraná - UTFPR - Ponta Grossa. guata@utfpr.edu.br

5 Professora titular da Universidade Tecnológica Federal do Paraná - UTFPR - Ponta Grossa. snasser@utfpr.edu.br
\end{abstract}


student for the activities carried out in Collabora, the better their performance was in the discipline, noting the collaborative learning efficiency.

Keywords: collaborative learning; academic performance; education of engineers.

\section{INTRODUÇÃO}

Uma pesquisa de doutorado, defendida em 2018, visou a responder o seguinte questionamento: "Quais contribuições que um Objeto Virtual de Aprendizagem Colaborativa, denominado Collabora, poderá trazer ao processo de ensino e aprendizagem na disciplina de Probabilidade e Estatística no Ensino Superior?" (ISHIKAWA, 2018).

A pesquisa consistiu na elaboração e aplicação do Collabora, Objeto Virtual de Aprendizagem Colaborativa (OVAC), capaz de avaliar a colaboração entre os pares e enfatizar aspectos pedagógicos e computacionais.

Os aspectos pedagógicos relacionam-se aos conceitos de design instrucional, como por exemplo: análise das necessidades de aprendizagem; concepção de um currículo ou programa; seleção e utilização de técnicas para determinar o conteúdo instrucional; planejamento da situação didática; e mapeamento e sequenciamento do conteúdo e preparação dos suportes - pedagógicos, tecnológicos e administrativos (ISHIKAWA, 2018).

Os aspectos computacionais, por sua vez, referem-se aos processos de desenvolvimento de softwares, específicos para área da computação. Para o desenvolvimento da tese, Ishikawa (2018) utilizou o modelo de desenvolvimento de software denominado "Protótipo evolutivo":

Protótipos evolutivos são criados nas fases iniciais do projeto e refinados ao longo do decorrer do processo de desenvolvimento do software, podendo ser interpretados como liberações. Incrementos de funcionalidade são incorporados ao protótipo, que, tendo sua fidelidade gradualmente aumentada, se torna o software final. Assim, os processos de desenvolvimento do protótipo e do software real são essencialmente o mesmo" (SOARES, p. 3, 2008).

Apoiado nesses aspectos pedagógicos e computacionais, o OVAC elaborado segue os princípios da Aprendizagem Colaborativa, e sua aplicação objetivou promover a aprendizagem na disciplina de Probabilidade e Estatística dos acadêmicos de um curso de Engenharia de Produção durante um semestre.

$\mathrm{Na}$ pesquisa de doutorado a preocupação foi a de verificar as contribuições para o ensino e aprendizagem a partir de uma análise qualitativa, baseada na Análise Textual Discursiva, em que, a partir das unidades e categorias que emergiram dos dados, os resultados apontaram para seis contribuições.

Entre essas contribuições, cita-se: proporcionou a interação durante a realização das atividades; ampliou o espaço da sala de aula e possibilitou que a aprendizagem ocorresse em diferentes ambientes; intuitivamente houve correlação positiva entre o nível de colaboração e o desempenho do acadêmico na disciplina; permitiu esclarecimento de dúvidas dos alunos sobre termos específicos dos conteúdos por meio de dicas; estimulou uma participação mais investigativa dos alunos durante as aulas e atividades presenciais em grupo; e provocou mudanças de concepções e atitudes, frente ao processo de ensino e aprendizagem na disciplina de Probabilidade e Estatística.

Buscando-se outros resultados, foi realizada uma análise quantitativa e para tanto analisou-se neste artigo a Correlação e Regressão Linear de duas variáveis discutidas na tese.

Nessa perspectiva, tem-se como objetivo deste artigo analisar a correlação entre as duas principais variáveis envolvidas na pesquisa de doutorado: o nível de colaboração e o desempenho do acadêmico na visão do professor. 
Com a finalidade de atender ao referido objetivo, as próximas seções esclarecem os pontos sobre a Aprendizagem Colaborativa Apoiada por Computador, a quantificação do nível de colaboração dos participantes a partir do Collabora, os procedimentos metodológicos aplicados neste artigo e resultados obtidos e discussões.

\section{APRENDIZAGEM COLABORATIVA APOIADA POR COMPUTADOR}

A Aprendizagem Colaborativa Apoiada por Computador (CSCL) tem como objetivo estudar o modo como se efetiva a aprendizagem em grupo com o auxílio do computador, aliada a um artefato que permite a interação entre entidades digitais, como por exemplo os Objetos Virtuais de Aprendizagem (GOMES; GADELHA; DE CASTRO, 2009).

Nessa perspectiva, durante uma pesquisa de doutorado do programa de Pós-Graduação em Ensino de Ciência e Tecnologia, da Universidade Tecnológica Federal do Paraná, em que foi elaborado e aplicado um Objeto Virtual de Aprendizagem denominado Collabora, os acadêmicos de Engenharia de Produção, envolvidos na aplicação da pesquisa, puderam "construir, interagir e colaborar com os seus pares, podendo criar estratégias para solucionar problemas durante a resolução dos exercícios" (ISHIKAWA, 2018, p. 15).

Essas ações são consideradas positivas para a aprendizagem acadêmica na disciplina de Probabilidade e Estatística conforme Cunha (2009), Cardoso (2010), Torres e Amaral (2011), Massaro (2014) entre outros pesquisadores que defendem a aprendizagem colaborativa promovida por meio de tecnologias.

De acordo com Torres (2007), a aprendizagem colaborativa está relacionada à promoção de uma aprendizagem mais dinâmica por meio do estímulo, do pensamento crítico, da capacidade de interação e da resolução de problemas. Esse tipo de aprendizagem possibilita um estreitamento das interações sociais entre os envolvidos na atividade e estimula o desenvolvimento da autonomia para a construção do conhecimento individual.

Oliveira (2008) complementa que para o êxito no processo colaborativo é imprescindível o empenho de cada um dos participantes na realização das tarefas. Para tanto, é importante que todos os participantes estejam motivados para colaborar em prol do todo, sem obstáculos para isso (STAHL et al., 2006).

Com o uso do Collabora, aplicado na tese de Ishikawa (2018), o professor pode gerenciar as atividades propostas no Objeto e acessar o desempenho de colaboração do indivíduo ou do grupo. No cenário colaborativo é fundamental a presença de instrumentos que tornem possível mensurar a colaboração de cada envolvido, de modo que seja viável identificar se houve colaboração e se os instrumentos contribuíram para a aprendizagem dos envolvidos.

O nível de colaboração é umas das chaves essenciais que torna realizável a análise da correlação entre a colaboração e o desempenho acadêmico, por isso a próxima seção esclarece sobre essa quantificação.

\section{QUANTIFICAÇÃO DO NÍVEL DE COLABORAÇÃO DOS PARTICIPANTES DO COLLABORA}

Para a obtenção do nível de colaboração, o Collabora efetua o cálculo de $\mathrm{C}$ (que representa o nível de colaboração), conforme equação abaixo (ISHIKAWA, 2018, p. 91):

$$
C=\frac{P_{1} \cdot\left(\frac{T P I}{T P G}\right)+P_{2} \cdot\left(\frac{T S I}{T S G}\right)}{\left(P_{1}+P_{2}\right)}
$$

Nessa equação (1) $\mathrm{P}_{1}$ refere-se ao peso atribuído às postagens; $\mathrm{P}_{2}$ ao peso atribuído às postagens consideradas significativas; TPG ao total de postagens de todos os integrantes do grupo6; TPI ao total de postagens do integrante do grupo; TSG ao total de postagens significativas de todos os integrantes do grupo; e TSI ao total de postagens significativas do integrante do grupo. 
Cabe ressaltar que os pesos atribuídos às postagens $(\mathrm{P} 1)$ e às postagens significativas (P2) foram definidos pelo professor e a soma das duas não é superior a 10,0. Foram consideradas postagens com valor ao processo colaborativo, por exemplo, links, arquivos e mensagens.

Conforme foi definido por Ishikawa (2018), a partir do valor de C, a colaboração do acadêmico pode ser classificada em 4 níveis de colaboração: Baixa colaboração; Média colaboração; Boa colaboração; e Muito boa colaboração (Quadro 1).

Quadro 1 - Faixas de valores para determinação de nível de colaboração

\begin{tabular}{|c|l|}
\hline Faixa de Valores para C & Nível de colaboração \\
\hline$\leq 0,25$ & Baixa colaboração \\
\hline$>0,25 \leq 0,5$ & Média colaboração \\
\hline$>0,5 e \leq 0,75$ & Boa colaboração \\
\hline$>0,75 e \leq 1,0$ & Muito boa colaboração \\
\hline
\end{tabular}

Fonte: Ishikawa, 2018.

A partir dos dados apresentados no Quadro 1, é possível visualizar, após efetivação do Cálculo de $\mathrm{C}$ dos acadêmicos, em qual nível de colaboração cada um se enquadra. Porém, nesta pesquisa, para a análise de correlação, foi considerado o valor numérico que representa o nível de colaboração de cada acadêmico.

Como desempenho do acadêmico, para esta pesquisa, considera-se o somatório das notas atingido pelo acadêmico nas avaliações escritas, corrigidas e pontuadas pelo professor da disciplina. A seção seguinte esclarece quanto aos procedimentos metodológicos para efetivação da análise.

\section{PROCEDIMENTOS METODOLÓGICOS}

Esta pesquisa é de caráter quantitativo, pois se envolve com análise de dados numéricos a partir da estatística descritiva e inferencial. Os dados utilizados foram extraídos por meio da aplicação do Collabora (ISHIKAWA, 2018), no decorrer de um semestre, em uma turma do $2^{\circ}$

7 A justificativa detalhada da escolha da disciplina e local de aplicação pode ser consultada em Ishikawa (2018), haja vista que os dados aqui utilizados estão apresentados em uma pesquisa de doutorado que período de Engenharia de Produção de uma Instituição de Ensino Superior Federal do Paraná, Brasil.

Participaram da aplicação 44 acadêmicos matriculados na disciplina de Probabilidade e Estatística no ano de 20177.

Para analisar a correlação entre o nível de colaboração e o desempenho do acadêmico na visão do professor, utilizou-se do conhecimento de Correlação e Regressão Linear. Antes de se efetivar a análise, os dados foram organizados em uma planilha eletrônica com o nível de colaboração dos alunos extraídos do Collabora e o desempenho acadêmico.

A Regressão Linear modela uma equação matemática de caráter linear que descreve o relacionamento de duas variáveis (TRIOLA, 2008). Existem vários usos dessas equações de regressão que visam a explicar a relação entre uma variável independente e outra dependente, ou seja, em que um determinado fenômeno (variável dependente) é consequência de outro (variável independente).

Segundo Triola (2008), o processo para encontrar uma equação linear matemática modela o relacionamento matemático entre as variáveis e visa a predizer valores futuros de uma variável dependente por meio de uma equação do tipo:

$$
\hat{y}=b_{0}+b_{1} x
$$

de tal modo que " $b_{0}$ " e " $b_{1}$ " são valores numéricos que podem ser encontrados por meio das equações (3) e (4):

$$
\begin{aligned}
b_{1} & =\frac{n\left(\sum x y\right)-\left(\sum x\right)\left(\sum y\right)}{n\left(\sum x^{2}\right)-\left(\sum x\right)^{2}} \\
b_{0} & =\bar{y}-b_{1} \bar{x}
\end{aligned}
$$

Nas equações (3) e (4), $y$ e $x$ representam respectivamente, nesta pesquisa, as notas de cada acadêmico na disciplina e o nível de colaboração, também de cada acadêmico. Assim, o somatório de $x y$ representa a soma da multiplicação de cada par ordenado, e $\bar{x}$ e $\bar{y}$ representam a média aritmética do conjunto de cada uma dessas variáveis.

enfatiza a importância do ensino de Probabilidade e Estatística para a formação de Engenheiros em uma perspectiva de aprendizagem colaborativa. 
Para certificar que a utilização da ferramenta de Regressão Linear realmente representa os dados, utiliza-se o método para encontrar o Coeficiente de Correlação de Pearson (TRIOLA, 2008), determinado pela equação:

$$
r=\frac{n \sum x y-\left(\sum x\right)\left(\sum y\right)}{\sqrt{n\left(\sum x^{2}\right)-\left(\sum x\right)^{2}} \sqrt{n\left(\sum y^{2}\right)-\left(\sum y\right)^{2}}}
$$

Nessa equação, $\mathrm{n}$ representa o tamanho da amostra, visto que o estudo se trata de uma análise estatística e selecionou para aplicação amostra - uma turma do curso de Engenharia de Produção.

Ainda, na equação (5), o coeficiente (r) resulta em valores entre -1 e 1 , em que o sinal indica se o relacionamento entre as variáveis é positivo ou negativo, enquanto $\mathrm{o}$ valor evidencia a força da relação entre elas, sendo assim uma correlação perfeita teria valor $1 \mathrm{ou}$ 1 e uma correlação zero significa que não existe correlação entre os dados (TRIOLA, 2008). A Figura 1 representa essa interpretação para $r$ :

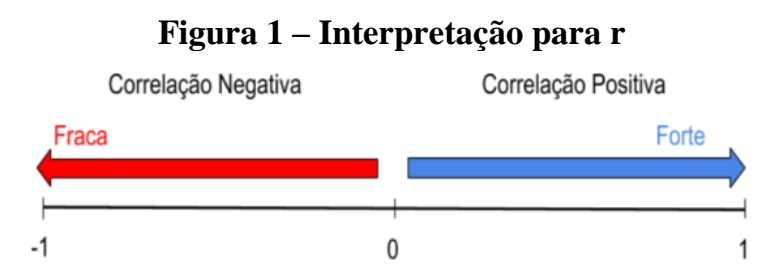

Fonte: acervo dos autores, 2019.

Em suma, a partir da Figura 1, quanto mais perto o coeficiente estiver de 1 (positivo ou negativo) maior é o grau de dependência estatística linear entre as variáveis, e quanto mais próximo de zero menor será a relação entre elas.

Uma correlação negativa forte indica uma dependência inversamente proporcional entre as variáveis, enquanto uma correlação positiva forte indica uma dependência diretamente proporcional. Isso significa que no caso de uma correlação negativa forte, à medida que a variável independente aumenta, por exemplo, a variável dependente diminuirá. Por sua vez, no caso de uma correlação positiva forte, ambas as variáveis acompanham um mesmo sentido, de crescimento ou decaimento.

No entanto, tais resultados extremos ( $1 \mathrm{ou}$ -1) dificilmente são alcançados em situações reais. Para comprovar que a estatística para a previsão do desempenho do aluno baseada em seu nível de colaboração possa representar toda a população de alunos que eventualmente utilize o Collabora, determina-se, se há ou não, uma correlação linear significativa entre duas variáveis, utilizando-se para tal o "Teste de Hipóteses para Correlação" (TRIOLA, 2008, p. 417).

$\mathrm{Na}$ aplicação desse Teste elaboram-se duas hipóteses: $H_{0}: \rho=0$ (a qual representa que não há correlação significativa) e $H_{1}: \rho \neq$ 0 (a qual representa correlação significativa). A mudança da variável $r$ para $\rho$ pode ser explicada por conta de que $r$ representa o coeficiente de correlação na amostra, e $\rho$ refere-se ao coeficiente de correlação para a população. Nesse sentido, em caso de aplicação do Teste e resultado de uma interpretação estatística de que há correlação significativa, será válido dizer que há correlação significativa aplicada à população e não somente à amostra (única turma de acadêmicos).

A interpretação estatística do teste visa a concluir "rejeição" ou "não rejeição" de Ho a partir do cálculo da estatística de teste representada pela equação (6),

$$
t=\frac{r}{\sqrt{\frac{1-r^{2}}{n-2}}}
$$

em que $r$ é o coeficiente de correlação linear explicitado anteriormente, $n$ é o tamanho da amostra e $t$ é a estatística de teste que determinará rejeição ou não da hipótese nula $\left(\mathrm{H}_{0}\right)$. O valor encontrado para $t$ é relacionado com um valor crítico disponível na tabela "Distribuição t", considerando um teste bilateral (TRIOLA, 2008) e localizado conforme está explicitado no Gráfico 2 na próxima seção.

A interpretação do Gráfico 1 é realizada a partir da representação do valor crítico (de modo a definir a região de rejeição ou não de $\mathrm{H}_{0}$ ), seguida da localização do valor $t$.

Além desse teste, para interpretar o coeficiente de correlação, esta pesquisa se apoia nos autores Dancey e Reidy (2006), que utilizam três categorias para classificar o coeficiente positivo de Pearson, explicitados no Quadro 2: 
Quadro 2 - Classificação do coeficiente de Pearson

\begin{tabular}{|c|l|}
\hline Valores de $\mathbf{r}$ & \multicolumn{1}{|c|}{ Classificação } \\
\hline $0,1 \leq \mathrm{r} \leq 0,3$ & Correlação fraca \\
\hline $0,4 \leq \mathrm{r} \leq 0,6$ & Correlação moderada \\
\hline $0,7 \leq \mathrm{r} \leq 1$ & Correlação forte \\
\hline
\end{tabular}

Fonte: acervo dos autores, 2019.

Ademais, segundo Triola (2008), é possível determinar também, a partir do coeficiente de correlação de Pearson, quanto da variação de $y$ pode ser explicado pela regressão linear calculada. Por exemplo, se $r=0,9$, o quadrado de $r$ (denominado coeficiente de determinação) será: $r^{2}=0,81$, o que significa que $81 \%$ da variação total de $y$ podem ser explicados pela reta de regressão (TRIOLA, 2008).

Nesse sentido, a partir desses conceitos estatísticos envoltos na Correlação e Regressão Linear, no coeficiente de Pearson e no Teste de Hipótese para Correlação, a próxima seção explicita e discute os resultados encontrados.

\section{RESULTADOS E DISCUSSÕES}

De acordo com o que foi apresentado nos procedimentos metodológicos, os dados foram organizados conforme a Tabela 1. Os acadêmicos foram codificados com a inicial A (acadêmico), seguidos de uma numeração de 1 a 44, a fim de se manter o sigilo quanto às suas identidades.

Tabela 1 - Organização dos dados

\begin{tabular}{|ccc|}
\hline Acadêmico & $\begin{array}{c}\text { Nível de } \\
\text { Colaboração }\end{array}$ & $\begin{array}{c}\text { Desempenho } \\
\text { acadêmico }\end{array}$ \\
\hline A.1 & 0,25 & 6,36 \\
A.2 & 0,33 & 4,07 \\
A.3 & 0,09 & 3,00 \\
A.4 & 0,23 & 5,43 \\
A.5 & 0,36 & 5,29 \\
A.6 & 0,00 & 0,00 \\
A.7 & 0,18 & 4,93 \\
A.8 & 0,14 & 4,50 \\
A.9 & 0,21 & 7,50 \\
A.10 & 0,09 & 3,14 \\
\hline
\end{tabular}

\begin{tabular}{|c|c|c|}
\hline A. 11 & 0,30 & 5,86 \\
\hline A. 12 & 0,26 & 7,00 \\
\hline A. 13 & 0,36 & 5,71 \\
\hline A. 14 & 0,38 & 0,36 \\
\hline A. 15 & 0,00 & 0,00 \\
\hline A.16 & 0,00 & 0,00 \\
\hline A. 17 & 0,20 & 3,93 \\
\hline A. 18 & 0,14 & 5,07 \\
\hline A. 19 & 0,02 & 1,21 \\
\hline A. 20 & 0,16 & 2,21 \\
\hline A. 21 & 0,18 & 0,00 \\
\hline A. 22 & 0,18 & 0,00 \\
\hline A. 23 & 0,05 & 2,71 \\
\hline A. 24 & 0,27 & 5,00 \\
\hline A. 25 & 0,41 & 7,50 \\
\hline A. 26 & 0,29 & 5,29 \\
\hline A. 27 & 0,29 & 7,29 \\
\hline A. 28 & 0,18 & 5,57 \\
\hline A. 29 & 0,20 & 3,14 \\
\hline A. 30 & 0,31 & 9,00 \\
\hline A. 31 & 0,29 & 6,43 \\
\hline A. 32 & 0,20 & 4,00 \\
\hline A. 33 & 0,16 & 4,93 \\
\hline A. 34 & 0,10 & 4,14 \\
\hline A. 35 & 0,22 & 5,14 \\
\hline A.36 & 0,23 & 8,79 \\
\hline A. 37 & 0,00 & 0,00 \\
\hline A. 38 & 0,21 & 4,00 \\
\hline A. 39 & 0,30 & 5,50 \\
\hline A. 40 & 0,26 & 6,00 \\
\hline A. 41 & 0,35 & 7,86 \\
\hline A. 42 & 0,25 & 6,07 \\
\hline A. 43 & 0,36 & 7,93 \\
\hline A. 44 & 0,21 & 4,79 \\
\hline
\end{tabular}

Fonte: elaborada pelos autores, 2019.

A partir desses dados (Tabela 1), definiuse o nível de colaboração como a variável independente $(x)$ e o desempenho acadêmico como a variável dependente $(\gamma)$.

Considerando todos os acadêmicos participantes da pesquisa (44) como tamanho da amostra (n), encontrou-se os resultados expostos no Gráfico 1. Os resultados plotados no gráfico foram calculados utilizando-se as funções estatísticas específicas (TRIOLA, 2008), os pontos representam a combinação das coordenadas do desempenho e da colaboração de cada aluno. A reta linear modela $o$ comportamento dos dados e é representada pela equação exposta no gráfico. 
Gráfico 1 - Representação dos resultados

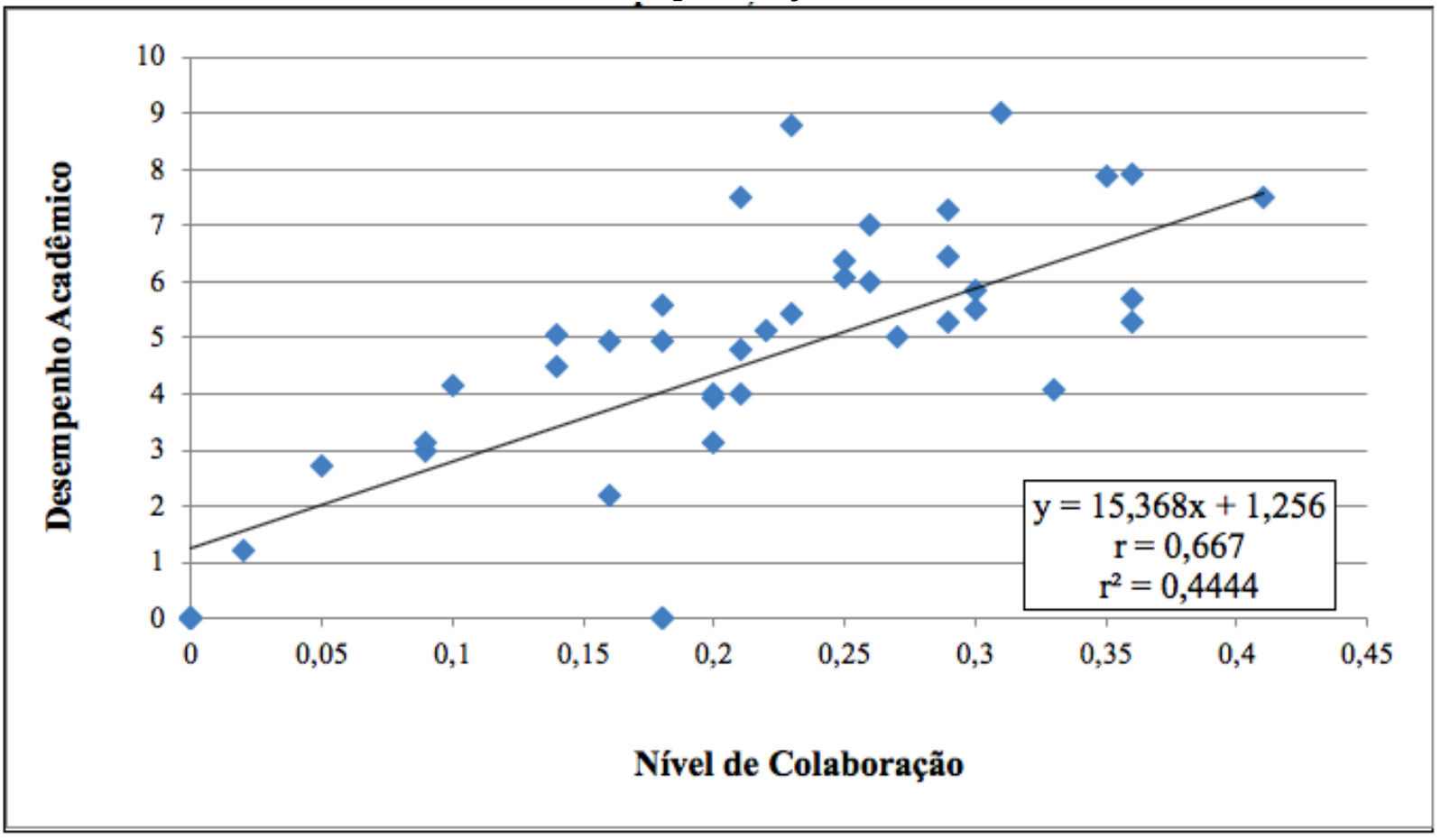

Fonte: elaborado pelos autores, 2019.

Conforme a representação, os resultados apontaram para um coeficiente de correlação (r) igual a 0,667. Conforme Triola (2008), é possível validar esse resultado a partir do Teste de Correlação (Equação 6).

Com a aplicação do Teste de Correlação, encontrou-se $t$ igual a 5,802.

A interpretação desse resultado indicou, com grau de confiança 99\%, correlação significativa dos dados. O valor crítico $(2,704)$ encontrado na tabela de "Distribuição $t$ " (TRIOLA, 2008) limitou a área de rejeição do teste bilateral, o que definiu a rejeição da hipótese nula $\left(\mathrm{H}_{0}\right)$ conforme representação no Gráfico 2.

Gráfico 2 - Representação dos resultados do Teste

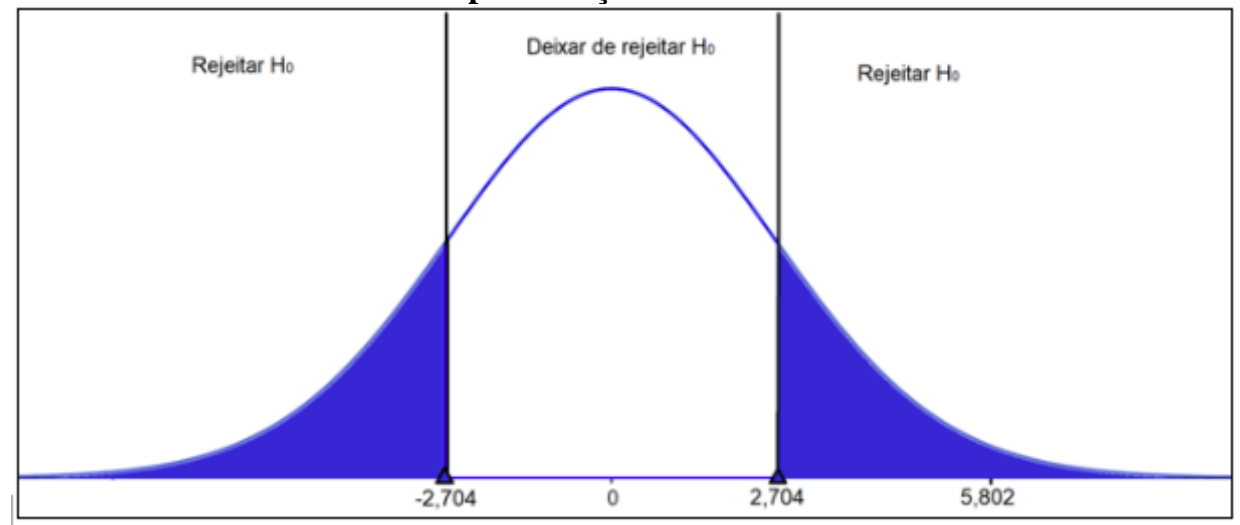

Fonte: elaborado pelos autores, 2019.

Dessa forma, fica validada, com 99\% de grau de confiança, a afirmação sobre a correlação significativa entre o nível de correlação e o desempenho acadêmico para toda a população de acadêmicos que venha a utilizar o Collabora.

Além do resultado do teste, considerando que o coeficiente de correlação encontrado 
pode ser arredondado para 0,7 , baseado na classificação de Dancey e Reidy (2006), há indicação para uma correlação positiva forte. Ademais, mesmo que se mantenha 0,6 , é possível afirmar sobre uma correlação positiva moderada segundo os mesmos autores. Isso é, há resultados estatísticos válidos para afirmarmos uma correlação positiva, classificada entre os graus moderada e forte, entre o nível de colaboração e desempenho acadêmico.

Essa correlação afirma que quanto maior o nível de colaboração, provavelmente o desempenho nas provas serão melhores. Assim sendo, corrobora-se Cunha (2009), Cardoso (2010), Torres e Amaral (2011) e Massaro (2014), pesquisadores esses que defendem a aprendizagem colaborativa tendo em vista a contribuição para a aprendizagem dos conteúdos.

A função matemática (reta de regressão) encontrada pelo processo da regressão linear a qual está descrita na Figura 1 (y = 15,368x + 1,256) - representa o modelo da correlação e, por meio dela, tendo-se a posse do nível de colaboração $(x)$ de um acadêmico, é possível estimar estatisticamente o seu desempenho acadêmico $(y)$.

Quanto a esse modelo, mediante ao coeficiente de determinação encontrado $\left(r^{2}=\right.$ 0,4444 ), é possível afirmar que $44 \%$ da variação total do desempenho acadêmico $(y)$ pode ser explicada pela reta de regressão. Neste sentido, $66 \%$ pode estar próximo da reta, visto que se trata de uma correlação classificada entre moderada e forte.

\section{CONSIDERAÇÕES FINAIS}

Em virtude dos resultados encontrados é possível sustentar a afirmação realizada intuitivamente por Ishikawa (2018). Desse modo, agora fica apresentada de maneira mais consistente a existência de correlação linear significativa, fundamentando-se em conceitos e métodos estatísticos.

Ainda, no que se refere à abordagem da aprendizagem colaborativa para Torres (2007), este estudo, aplicado ao ensino de Engenharia, possui três principais objetivos: a transformação do professor em um facilitador, o desenvolvimento de habilidades de metacognição e o aumento da aprendizagem por meio da colaboração entre os alunos.

Trazendo-se à luz tais objetivos, entendese com os resultados encontrados neste artigo que aumentar a aprendizagem por meio da colaboração é algo possivelmente atendido na utilização do ambiente virtual Collabora, tendo em vista que o desempenho do acadêmico está, segundo os resultados encontrados, diretamente relacionado a sua colaboração de modo significativo.

Por fim, ressalta-se a eficiência da aprendizagem colaborativa aplicada na formação de engenheiros, que, neste estudo, contribui e se centra na disciplina de Probabilidade e Estatística, porém explicita dados estatísticos favoráveis para futuras aplicações para outras disciplinas dos cursos de Engenharia.

\section{REFERÊNCIAS}

\section{CARDOSO, A. L. M. S. Construção e difusão} colaborativa do conhecimento: uma experiência construtivista de educação em um ambiente virtual de aprendizagem. 2010. Tese (Doutorado em Educação) Universidade Federal da Bahia, Salvador, 2010.

CUNHA, A. A. S. Definição de um modelo de sistema de aprendizagem colaborativa apoiado por computador com ênfase em trabalho em equipe. 2009. Dissertação (Mestrado em Engenharia Elétrica e de Computação) - Universidade Estadual de Campinas. Campinas, 2009.

DANCEY, C.; REIDY, J. Estatística sem matemática para psicologia: usando SPSS para Windows. Porto Alegre, Artmed, 2006.

GOMES, S. R; GADELHA, B. F.; DE CASTRO, A. N. Objetos de Aprendizagem Funcionais: Uma Abordagem Prática. Anais... Simpósio Brasileiro de 
Informática na Educação. XX, Florianópolis, UFSC-SC, 2009.

ISHIKAWA, E. C. M. Objeto virtual de aprendizagem colaborativa (Collabora): estudo na disciplina de probabilidade e estatística no ensino superior. 2018. Tese (Doutorado em Ensino de Ciência e Tecnologia) - Universidade Tecnológica Federal do Paraná, Ponta Grossa, 2018.

MASSARO, G. Recursos Educacionais abertos e aprendizagem colaborativa: novas perspectivas na construção e utilização de materiais educacionais. Colabor@-A Revista Digital da CVA-RICESU, v. 8, $\mathrm{n}$. 31, 2014.

OLIVEIRA, E. A. Um modelo de colaboração inteligente personalizada para ambientes de EAD. 2008. Dissertação (Mestrado em Ciência da Computação) Centro de Informática, Universidade Federal de Pernambuco, Recife, 2008.
SOARES, B. C. Requisitos para utilização de prototipagem evolutiva nos processos de desenvolvimento de software baseado na Web. Belo Horizonte: UFMG, 2008.

STAHL, G. et al. Computer-supported collaborative learning: An historical perspective. Cambridge handbook of the learning sciences, 2006.

TORRES, P. L. Aprendizagem colaborativa. Algumas vias para entretecer o pensar e o agir. Curitiba: SENAR-PR, 2007.

TORRES, T. Z.; DO AMARAL, S. F. Aprendizagem Colaborativa e Web 2.0: proposta de modelo de organização de conteúdos interativos. Educação Temática Digital, v. 12, p. 49, 2011.

TRIOLA, M. Introdução à Estatística. Rio de Janeiro: LTC, 2008.

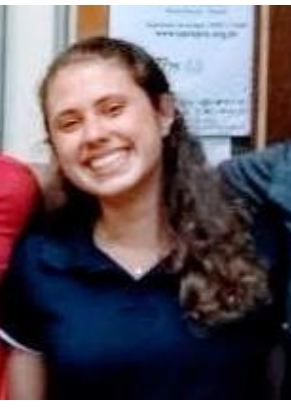

\section{DADOS BIOGRÁFICOS DOS AUTORES}

Julia Eunice Fernandes - Graduanda em Engenharia de Produção e aluna de Iniciação Científica do Programa de Ensino de Ciência e Tecnologia, bolsista CNPq, da Universidade Tecnológica Federal do Paraná - UTFPR. Contato: juliafernandes@alunos.utfpr.edu.br.

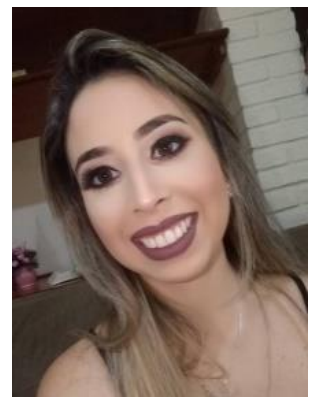

Caroline Subirá Pereira - Graduação em Matemática (Licenciatura) pela Universidade Estadual do Norte do Paraná (2012), Especialista em Ensino de Matemática pela Faculdade Cristo Rei - FACCREI (2013), Graduação em Pedagogia (Licenciatura) pelo Instituto de Educação Superior Alvorada Plus (2013), Graduação em Física (Licenciatura) pela Universidade Metropolitana de Santos (2015) e Mestra em Ensino de Ciência e Tecnologia pela Universidade Tecnológica Federal do Paraná - UTFPR (2017). Atualmente é Doutoranda do Programa de PósGraduação em Ensino de Ciência e Tecnologia, bolsista CAPES, da Universidade Tecnológica e Federal do Paraná, Ponta Grossa, Paraná, Brasil. Contato: carolinepereira@alunos.utfpr.edu. 

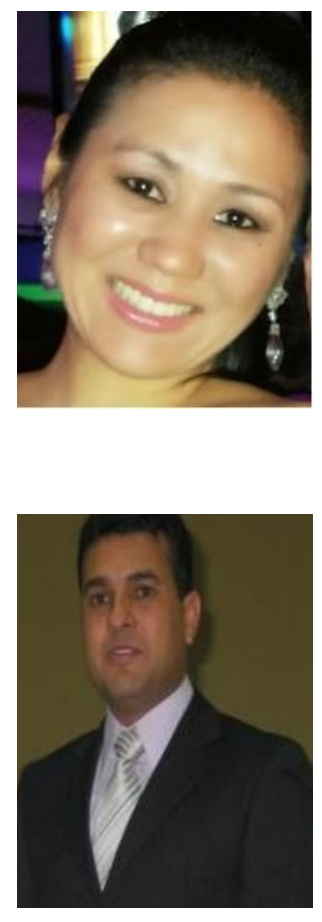

Guataçara dos Santos Junior - Graduação em Matemática (Licenciatura) pela Universidade Estadual de Ponta Grossa (1993), Mestrado em Ciências Geodésicas pela Universidade Federal do Paraná (2001) e Doutorado em Ciências Geodésicas pela Universidade Federal do Paraná (2005). Atualmente é professor na Universidade Tecnológica Federal do Paraná - Câmpus Ponta Grossa. Atua na Graduação com a disciplina Probabilidade e Estatística para cursos de Engenharia. Também atua na pós-graduação: Programa de Pós-Graduação em Ensino de Ciência e Tecnologia, cursos de mestrado e doutorado, onde orienta e trabalha com a disciplina Tópicos de Estatística Aplicada. Contato: guata@utfpr.edu.br.

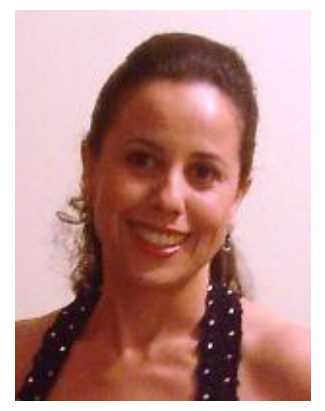

Simone Nasser Matos - Graduada em Bacharel em Processamento de Dados pela Universidade Estadual de Ponta Grossa (1993), Mestre em Inteligência Computacional pela Universidade Federal do Paraná (2001) e Doutora em Ciências pelo Instituto Tecnológico de Aeronáutica (2008). Atualmente é professora titular da Universidade Tecnológica Federal do Paraná, Ponta Grossa, Paraná, Brasil. Contato: snasser@utfpr.edu.br. 